\title{
APROXIMACIÓN DE LA CALIDAD DE VOZ Y COBERTURA EN UNA RED GSM DE EMERGENCIA
}

\section{SURVEY ANALYSIS OF VOICE QUALITY AND COVERAGE IN A GSM EMERGENCY NETWORK}

\author{
Elvis Eduardo Gaona García', Miguel Antonio Ávila Angulo², Elkin Gabriel Muskus ${ }^{3}$
}

Fecha de recepción: 28 de enero de 2014

Fecha de aprobación: 24 de julio de 2014

Referencia: E.E. Gaona García, M.A. Ávila Angulo, E.G. Muskus Rincón. (2014). Aproximación de la calidad de voz y cobertura en una red GSM de emergencia. Ciencia e Ingeniería Neogranadina, 24 (2), pp. 23 - 36

\section{RESUMEN}

En este documento se muestran los resultados obtenidos al medir los parámetros de calidad de voz en una solución propuesta de telecomunicaciones para la atención de desastres en situaciones de emergencia, haciendo uso de software libre para interconectar llamadas, protocolos de comunicaciones empleados en las redes GSM (Global System Mobile) en la banda DCS-1800 (Digital Cellular Service - 1800MHz), que facilita el uso de cualquier terminal móvil comercial, utilizando un USRP (Universal Software Radio Peripheral) en conjunto con sistema de telefonía IP basado en Asterisk. El USRP se encarga de emular la operación de una BTS (Base Transceiver Station) al ejecutar las funciones de un PBX (Private Branch Exchange), permitiendo la coordinación de las entidades responsables de responder y atender este tipo de emergencias. Las mediciones del desempeño de la red se realizaron a partir de tres parámetros probabilísticos: GoS (Grade of service), DCR (Dropped Call Rate) y MOS (Mean Opinion Score). Con el uso de 8 terminales y escogiendo un GoS del 2\% se generó un tráfico de hasta 3,6 Erlang. Esta medición se realizó empleando modelos probabilísticos de Erlang B. Se realiza el comparativo de la cobertura de la red aplicando el modelo Okumura-Hata sin el uso de amplificadores.

Palabras clave: Calidad de voz, cobertura, modelos de propagación, telefonía móvil celular, Dropped rate.

\footnotetext{
1. Ingeniero Electrónico, Ms.C., Facultad de Ingeniería, Docente y director del grupo de investigación GITUD. Universidad Distrital Francisco José de Caldas, Bogotá, Colombia, egaona@udistrital.edu.co; elvisgaona@ieee.org

2. Ingeniero Catastral y Geodesta, Ms.C., Facultad de Ingeniería, Docente. Universidad Distrital Francisco José de Caldas, Bogotá, Colombia, maavila@udistrital.edu.co

3. Ingeniero Electrónico, Ms.C., Facultad de Ingeniería, Docente e investigador del grupo de investigación GITUD. Universidad Distrital Francisco José de Caldas, Bogotá, Colombia, egmuskusr@udistrital.edu.co
} 


\section{ABSTRACT}

This paper contains the results obtained by measuring the voice quality parameters in a mobile telecommunications network for disaster attention in an emergency situation. The infrastructure used free software, an IP telephony system based on Asterisk, to interconnect calls, also used communications protocols of GSM (Global System Mobile) in the DCS-1800 (Digital Cellular Service band - $1800 \mathrm{MHz}$ ) that facilitates the use of any commercial mobile terminal. The system also used USRP (Universal Software Radio Peripheral), that is responsible for emulating the operation of a BTS (Base Transceiver Station) performing the functions of a PBX (Private Branch Exchange), further allows the coordination of different entities in charge of responding to these type of emergencies. The measurements of network performance were made from three probabilistic parameters: GoS (Grade of Service), DCR (Dropped Call Rate) and MOS (Mean Opinion Score). Using eight mobile stations and choosing a GoS of 2\%, traffic up to 3.6 Erlang was generated. This measurement was performed using probabilistic models of Erlang B. Comparison the coverage of the BTS is carried out by applying the model OkumuraHata without amplifiers.

Keywords: Voice quality, coverage, propagation model, cellular mobile telephony, Dropped rate.

\section{INTRODUCCIÓN}

Varios estudios que exploran el uso de herramientas de software libre GNU [1-3] reportan las experiencias del funcionamiento de una red GSM utilizando tarjetas hijas con una unidad USRP, al igual que la implementación de este tipo de sistemas en zonas rurales [4-8], lo que muestra varias ventajas al tener un sistema de bajo costo en lugares de difícil acceso sin presencia de operadores móviles.

Otros estudios [9-11] muestran que el uso de BTS basada en software como sistema embebido ha ido en aumento con la implementación de pico celdas. Bai et al. [12] han dado soluciones al problema de comunicaciones en situaciones de emergencia, utilizando redes WMN (Wireless Mesh Network) donde se emplean saltos entre dos o más nodos. Ochoa y Santos [13] logran un área de cobertura determinada, que aumenta la eficiencia y la eficacia del proceso de respuesta de emergencia. El rango de cobertura de los sistemas WMN es limitado debido a que en cada salto entre nodos se generan pérdidas y/o retardos (latencias) de los datos, así su cobertura no es netamente proporcional al número de nodos usados. Song et al. [14] muestran que la cobertura de estos sistemas puede alcanzar los 300 m por salto, con retardos de hasta 100 ms en 10 saltos. Lien et al. [15] proporcionan resultados de la cobertura alrededor de $21 \mathrm{~m}$ por salto con retardos de 641 ms, medida de MOS de 2 y una tasa de perdida promedio de $35,5 \%$ en sistemas tipo walkie-talkie. El alcance aumenta en [16] a $24 \mathrm{~m}$ con un tiempo de retardo promedio de 1018 ms, una tasa promedio de pérdidas del $11 \%$ y un MOS de 2 a 3. Para las redes ad hoc las pruebas realizadas en [17-18] muestran la fiabilidad del sistema con una cantidad reducida de nodos. Gadallah y Serhani [19] muestran el aumento en el manejo de peticiones utilizando una MANET y adaptando las técnicas de servicio sobre diferentes escenarios. 


\section{ARQUITECTURA DE LA RED GSM}

\subsection{CENTRAL DE CONMUTACIÓN}

Al utilizar el USRP se cumple con uno de los requerimientos del sistema de conmutación, como lo es su portabilidad y bajo costo. Asimismo, el sistema ha de cumplir con las principales funciones que se desempeñan en una red móvil celular desde las estaciones base o BTS.

- PBX Asterisk: Asterisk es un software que implementa algunas funcionalidades de centrales telefónicas conocidas como PBX bajo licencia GPL (Licencia Pública General de GNU, por sus siglas en inglés). Permite su implementación sobre sistemas operativos Linux [20], aspecto que reduce notablemente el costo de implementación [21].

- OpenBTS: Aplicación que se encuentra en constante desarrollo. Pretende implementar y desarrollar sistemas basados en USRP por medio de aplicaciones Unix de código abierto haciendo uso de sistemas libres de conmutación o PBX para establecer comunicaciones mediante la interface aérea GSM ("Um") con dispositivos móviles [6], y es lo suficientemente robusta para igualar la operatividad de una BTS estándar. En la Figura 1 se presenta la arquitectura general de este punto de acceso llamado OpenBTS.

\subsection{MODULADOR}

El modulador es la interfaz que toma los datos y la información encriptada dentro del protocolo del estándar GSM y la modula a la frecuencia portadora deseada (para el caso $1800 \mathrm{MHz}$ ). No se realiza amplificación de la señal, solo se modula buscando su compatibilidad con la banda GSM. Este tipo de sistemas deben emplear software libre como GNU Radio que les permite ser mediadores entre software y hardware, para este caso se emplea como medio de comunicación el puerto USB.

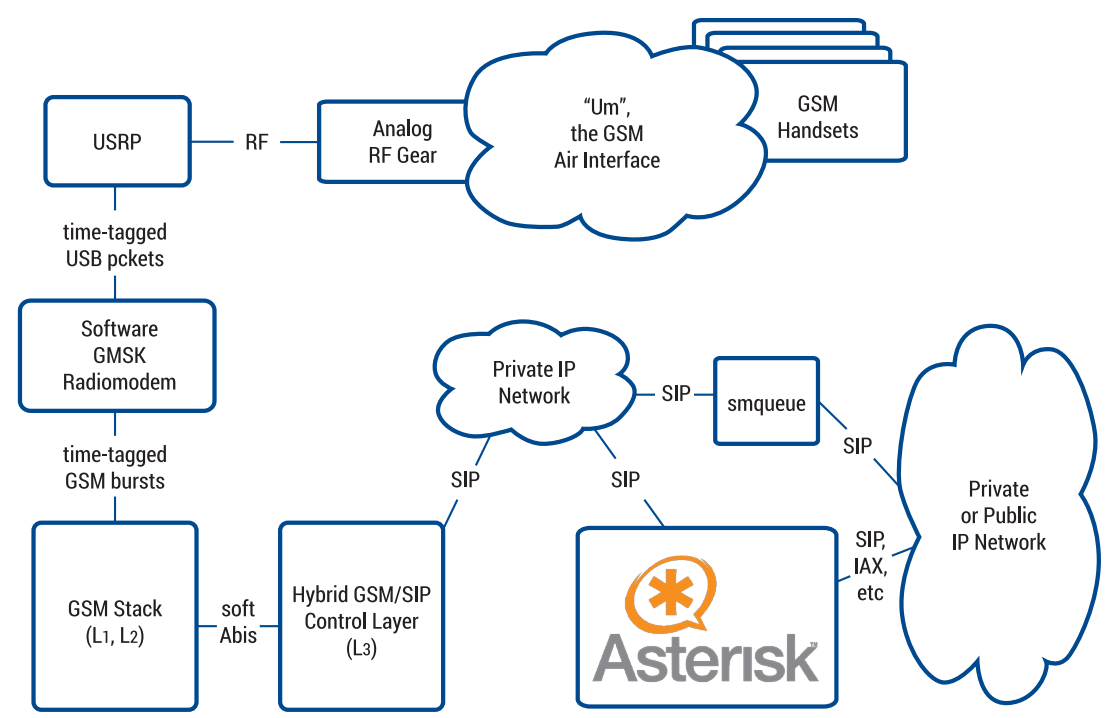

Figura 1. Arquitectura OpenBTS.

Fuente: [22] 


\subsubsection{USRP}

Permite transmitir y recibir datos modulados. El modelo USRP1 se encuentra dentro de la gama media de USRP de Ettus Research [23], y recibe y transmite las Ilamadas simultáneamente en tiempo real. El sistema embebido en el USRP solo se ocupa del procesamiento en alta frecuencia y de transmitir y recibir información empaquetada al procesamiento de baja frecuencia que ejecuta por medio software. Para esto se utiliza una tarjeta transceptora que opera en la banda 1,5-2,1 GHz (RFX1800). La frecuencia utilizada se da en la banda DCS-1800 por razones de congestión e interferencia en esta banda, evitando así conflictos legales con los demás operadores prestadores del servicio.

\subsubsection{GNU Radio}

Este software libre está desarrollado en lenguajes de programación Python y $\mathrm{C}++$ y provee las herramientas necesarias para el procesamiento de señales en sistemas de radio en $\mathrm{RF}$.

\subsection{ESTACIÓN MÓVIL}

La estación móvil se utiliza como intercomunicador final. En este caso se aplica el término a los terminales móviles, habilitando el uso de cualquier terminal móvil que funcione en la banda de $1800 \mathrm{MHz}$.

El esquema básico de un sistema GSM se muestra en la Figura 2. Con él se realizan las pruebas de corto alcance y se comprueba la conexión del sistema basado en software, así como el transceptor universal USRP1, la antena y la compatibilidad con los componentes y el dispositivo móvil.

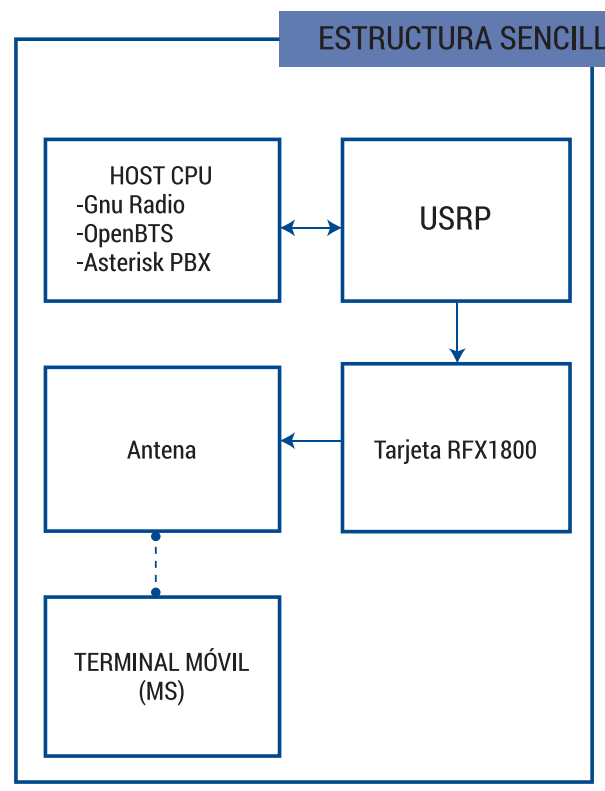

Figura 2. Esquema básico del sistema GSM.

\section{DIMENSIONAMIENTO DE LA RED GSM DE EMERGENCIA}

La planeación de la red se reduce a la implementación de una pico-celda. En la planeación y dimensionamiento del sistema de comunicaciones se realizaron las consideraciones de un enlace de microondas con línea de vista estándar, obteniendo las pérdidas totales de los enlaces (Pérdidas Isotrópicas del Enlace) y aplicando este cálculo a un modelo estándar de propagación, buscando así obtener la cobertura del sistema.

\subsection{CRITERIOS DE PLANIFICACIÓN}

En esta etapa se tienen en consideración los parámetros de calidad y cobertura de la red a implementar, que tienen lugar antes de la planificación de la red. El límite de potencia de la señal que determina el cubrimiento está dado por una potencia recibida de $-75 \mathrm{dBm}$. 
Se estiman tres parámetros probabilísticos que determinan la calidad de la red: en primer lugar, se considera la tasa de llamadas exitosas mayor al 98\%; en el caso de la tasa de llamadas rechazadas, se establece un $2 \%$ (de cada 100 Ilamadas se bloquean 2); mientras que para el MOS, que evalúa la calidad de la llamada, debe ser mayor a 3,5 en una escala de 0 a 5 .

\subsection{PARÁMETROS DE LOS DISPOSITIVOS DE LA RED}

En el dimensionamiento se requieren conocer parámetros de los dispositivos involucrados como potencia del transmisor, ganancia de las antenas, pérdidas del cable en la línea de transmisión, sensibilidad del receptor, entre otros. La Tabla 1 recopila una explicación de los parámetros usados en el cálculo de este presupuesto de potencia.

\subsubsection{Estación Móvil}

La estación móvil hace uso de dispositivos móviles comerciales en uso con otro operador.
Sus características son similares, ya que adoptan los estándares impuestos por medio del estándar GSM.

Los datos a indagar sobre esta antena receptora constan de tres parámetros: la potencia transmitida por la MS (Mobile Station), la ganancia de la antena receptora y la sensibilidad del receptor. Según el estándar GSM 05.05 de la ETSI [24], el nivel de referencia de sensibilidad que no puede sobrepasarse dependiente del tipo de canal usado y las condiciones de propagación está limitado a - 100dBm para DSC1800 clase 1 y 2 , y $-102 \mathrm{dBm}$ para clase 3 . La producción actual de dispositivos móviles celulares se desarrolla para clase 1 en la banda 1800, con lo que se tiene:

$$
S_{M S}=-100 \mathrm{dBm}
$$

La potencia transmitida de la MS tiene un valor máximo de $36 \mathrm{dBm}$, mientras que el valor típico es de $25 \mathrm{dBm}$ dependiendo de la clase

Tabla 1. Descripción de los parámetros del presupuesto de potencia.

\begin{tabular}{|c|l|}
\hline PARÁMETRO & $\begin{array}{l}\text { DESCRIPCIÓN } \\
\text { en } \mathrm{dBm} \text {. }\end{array}$ \\
\hline$P_{T}$ & $\begin{array}{l}\text { Potencia de transmisión de la antena. En conjunto con la ganancia de la antena } \\
\text { forman la PIRE (Potencia Isotrópica Radiada Equivalente) del transmisor en W } \\
\text { (Vatios). }\end{array}$ \\
\hline$G$ & Ganancia de la antena transmisora-receptora. \\
\hline$G_{D}$ & $\begin{array}{l}\text { Ganancia por diversidad. Ésta ganancia comúnmente se usa para balancear el } \\
\text { enlace de bajada con el de subida y se ubica en el enlace de recepción de la BTS; } \\
\text { usualmente se emplean 5dB para esta ganancia. }\end{array}$ \\
\hline$L_{L T}$ & $\begin{array}{l}\text { Pérdidas de la línea de transmisión que incluyen las pérdidas totales del medio } \\
\text { conductor empleado en conjunto con los conectores. }\end{array}$ \\
\hline$L_{D}$ & Pérdidas del duplexor. \\
\hline$L_{B P F}$ & Pérdidas del filtro pasa banda. \\
\hline$M_{D I}$ & Margen de degradación por interferencia. \\
\hline
\end{tabular}


de alimentación. El valor estándar de control para cualquier clase de MS es de OdBm al igual que la ganancia estándar de las antenas receptoras, así se tiene:

$$
\begin{gathered}
P_{T M S}=25 d B m \\
G_{M S}=0 d B
\end{gathered}
$$

\subsubsection{Estación Transceptora Base (BTS)}

La potencia de transmisión de la USRP1 en conjunto con las tarjetas RFX1800 es de
$100 \mathrm{~mW}(20 \mathrm{dBm})$ y una figura de ruido NF (Noise Factor) de 8dB [5]. La sensibilidad recomendada de las BTS según la norma 05.05 de la ETSI es de $S=-106 \mathrm{dBm}$. De esta forma se calcula la sensibilidad de la USRP1 utilizando la figura de ruido y la relación mínima de Eb/ NO de 8dB definida en la norma ETSI 05.05. La ecuación que determina la sensibilidad se da a continuación:

$$
S_{B T S}=\frac{E_{b}}{N_{0}} F k T_{0} W
$$

donde $\mathrm{k}$, es la constante de Boltzman, To=290K y el ancho de banda $W=271 \mathrm{KHz}$, $(54 \mathrm{dBHz})$.

$$
\begin{aligned}
S_{B T S} & =8 d B+8 d B+(290 K \times 1,38 \times 10 \quad J / K)+54 d B H z \\
S_{B T S} & =8 d B+8 d B-174 d B m+54 d B H z=-104 d B m \\
P_{T B T S} & =20 d B m
\end{aligned}
$$

\subsubsection{Antena}

La ganancia de la antena tanto para la estación transmisora y el receptor móvil es el de una antena tipo dipolo de $3 \mathrm{dBi}$.

$$
G_{B T S}=3 d B i
$$

\subsection{PRESUPUESTO POTENCIA Y COBERTURA}

El cálculo del presupuesto de potencia se realiza para el enlace de subida y de bajada mostrados en la Tabla 2, esto se proporciona con el fin de balancear los enlaces. En el procedimiento de cálculo del presupuesto de potencia el modelo se plantea sin el uso de amplificadores, duplexores o filtros, estos valores se toman iguales a cero. Las pérdidas a lo largo de la trayectoria del enlace (LTT) se derivan de los diferentes efectos físicos que lo afectan. En la Tabla 2 se obtiene el valor total para el sistema implementado, obteniendo (7).

$$
L_{T T}=116,87 d B
$$

Para el cálculo de la cobertura del enlace de comunicaciones se emplean los siguientes modelos de propagación.

\subsection{MODELO OKMURA-HATA}

Este modelo cubre el rango de frecuencias $150 \mathrm{MHz}$ a $1920 \mathrm{MHz}$ incluyendo el tipo de terreno. La atenuación está regida por la ecuación (8) cuyos parámetros se describen en la Tabla 3. 
El término de correlación se emplea según el tipo de terreno donde se realiza su implementación. En este caso se trabaja terreno urbano y se emplea (9), donde $h_{r}$ es la altura de la MS, estimada de ser en promedio de $1,6 \mathrm{~m}$.
Al resolver la ecuación (8) e incluir el valor de c calculado en (9), la altura de la antena de la BTS de $5 \mathrm{~m}$ de altura (máximo) y las pérdidas de trayectoria dadas por el presupuesto de potencia en (7) a =LTT se resuelve el valor de la cobertura del enlace con el valor de d.

Tabla 2. Procedimiento de cálculo del presupuesto de potencia para el sistema comercial.

\begin{tabular}{|c|c|c|c|c|}
\hline $\begin{array}{l}\text { PRESUPUESTO DE POTENCIA DEL } \\
\text { RADIO ENLACE }\end{array}$ & \multicolumn{4}{|c|}{ MS CLASE 1} \\
\hline Frecuencia [MHZ]: & 1800 & \multirow[b]{2}{*}{ BTS } & \multicolumn{2}{|c|}{ SISTEMA: GSM } \\
\hline Terminal receptora & UNIDAD & & MS & \\
\hline Sensibilidad RF de entrada del RX & $\mathrm{dBm}$ & -104 & -100 & A \\
\hline $\begin{array}{l}\text { Margen de degradación por } \\
\text { interferencia }\end{array}$ & $d B$ & 5 & 5 & B \\
\hline Pérdidas del cable + conectores & $\mathrm{dB}$ & 1,86 & 0 & C \\
\hline Ganancia antena del RX & $\mathrm{dBi}$ & 3 & 0 & $\mathrm{D}$ \\
\hline Ganancia por diversidad & $\mathrm{dB}$ & 5 & 0 & $\mathrm{E}$ \\
\hline Potencia isotrópica & $\mathrm{dBm}$ & $-105,14$ & -95 & $\begin{array}{l}F=A+B+C- \\
D-E\end{array}$ \\
\hline Fuerza del campo & $\mathrm{dB} \mu \mathrm{V} / \mathrm{m}$ & 36,9854 & 47,1254 & $\mathrm{G}=\mathrm{F}+\mathrm{Z}$ \\
\hline Terminal transmisora & UNIDAD & MS & BTS & \\
\hline Potencia RF pico de salida del TX & W & 0,316 & 0,1 & \\
\hline Potencia media por ciclo de RF & $\mathrm{dBm}$ & 25 & 20 & $\mathrm{~K}$ \\
\hline Duplexor + filtro & $\mathrm{dB}$ & 0 & 2,4 & $L$ \\
\hline $\begin{array}{l}\text { Potencia RF pico de la salida del } \\
\text { combinador }\end{array}$ & $\mathrm{dBm}$ & 25 & 17,6 & $M=K-L$ \\
\hline Pérdidas del cable + conectores & $\mathrm{dB}$ & 0 & 1,13 & $\mathrm{~N}$ \\
\hline Ganancia de antena de TX & $\mathrm{dBi}$ & 0 & 3 & 0 \\
\hline EIRP & $\mathrm{dBm}$ & 25 & 19,47 & $\mathrm{P}=\mathrm{M}-\mathrm{N}+\mathrm{O}$ \\
\hline Pérdidas isotrópicas del trayecto & $\mathrm{dB}$ & 130,14 & 114,47 & $\mathrm{Q}=\mathrm{P}-\mathrm{F}$ \\
\hline \multicolumn{5}{|l|}{ Enlace de bajada } \\
\hline $\begin{array}{l}\text { Potencia pico de salida del TX de la } \\
\text { BTS }\end{array}$ & $\mathrm{dBm}$ & 35,14 & $P=Q+F$ & \\
\hline Potencia media por ciclo de RF & $\mathrm{dBm}$ & 35,67 & \multicolumn{2}{|c|}{$\mathrm{K}=\mathrm{M}+\mathrm{L}=\mathrm{P}+\mathrm{N}-\mathrm{O}+\mathrm{L}$} \\
\hline
\end{tabular}

Fuente: Cárdenas, 2011 [25]. 
Tabla 3. Descripción de la ecuación (8) del modelo Okmura-Hata

\begin{tabular}{|c|l|}
\hline PARÁMETRO & \multicolumn{1}{|c|}{ DESCRIPCIÓN } \\
\hline$a$ & Perdidas en la trayectoria (dB). \\
\hline$f$ & Frecuencia en GHz. \\
\hline$H_{e f f}$ & Altura a la que se ubica la antena de la BTS en m. \\
\hline$c$ & $\begin{array}{l}\text { Término de correlación que depende del terreno; en este caso se da por } \\
{[25] .}\end{array}$ \\
\hline$d$ & Distancia entre BTS y MS (alcance) en $\mathrm{m}$. \\
\hline
\end{tabular}

$$
\begin{aligned}
& c=(1,1 \log (f)-0,7) h_{r}-(1,56 \log (f)-0,8) \\
& c=0,331055 d B
\end{aligned}
$$

\subsection{MODELO WALFISCH-IKEGAMI}

Cubre la banda UHF de corto alcance [26]. La cobertura se calcula para condiciones ideales de línea de vista del modelo Walfisch-Ikegami (WI) regido por la ecuación (10). Esta ecuación no contempla las consideraciones de difracción de la señal debido al terreno y sus parámetros se explican en la Tabla 4.

$$
a=42,6+26 \log (d)+20 \log (f)
$$

\subsection{MODELO FRIIS}

También se considera la cobertura utilizando el modelo de propagación ideal de Friis (F) regido por la ecuación (11), cuyos parámetros se explican en la Tabla 5.

$$
a=92,4+20 \log (d)+20 \log (f)
$$

Los resultados de los anteriores modelos se muestran en la Tabla 6, en la que se comparan

Tabla 4. Descripción de la ecuación (10) del modelo Walfisch-Ikegami.

\begin{tabular}{|c|l|}
\hline PARÁMETRO & \multicolumn{1}{c|}{ DESCRIPCIÓN } \\
\hline$a$ & Perdidas en la trayectoria (dB). \\
\hline$f$ & Frecuencia en MHz. \\
\hline$d$ & Distancia entre BTS y MS (alcance) en Km. \\
\hline
\end{tabular}

Tabla 5. Descripción de la ecuación (11) del modelo Friis.

\begin{tabular}{|c|l|}
\hline PARÁMETRO & \multicolumn{1}{|c|}{ DESCRIPCIÓN } \\
\hline$a$ & Perdidas en la trayectoria (dB). \\
\hline$f$ & Frecuencia en GHz. \\
\hline$d$ & Distancia entre BTS y MS (alcance) en Km. \\
\hline
\end{tabular}


Tabla 6. Resultados de la cobertura con los modelos de propagación

\begin{tabular}{|l|c|}
\hline Modelo de Propagación & Distancia d $(\mathrm{Km})$ \\
\hline Okumura-Hata & 0,018018 \\
\hline Walfisch-Ikegami & 2,25156 \\
\hline Friis & 9,29453 \\
\hline
\end{tabular}

con las mediciones obtenidas en sitio para el modelo Okumura-Hata. Este modelo incluye las condiciones de las zonas urbanas, de interiores como edificios y materiales de construcción que son las condiciones bajo las cuales se realizaron las mediciones. El cálculo de cobertura muestra un alcance de $18 \mathrm{~m}$. De esta forma la cobertura está dada mucho más cercana a la realidad que la de espacio libre de los modelos de Friis y Walsfisch-Ikegami [27-28].

\section{RESULTADOS}

\subsection{ALCANCE Y COBERTURA}

La distancia del enlace se midió manteniendo una llamada de prueba entre una MS y el SoftPhone (SP), hasta interrumpir la comunicación. En la Tabla 7 se muestran los resultados.

La cobertura se determinó con el nivel de señal recibida entre los $-75 \mathrm{dBm}$ a -77dBm según el estándar GSM para zonas urbanas en interiores [24]. Con la aplicación en el terminal móvil "Antenas para Android" se midió este valor en distintas direcciones y sus resultados se muestran en la Tabla 8, que evidencian la estabilidad en el área de cobertura de forma radial con la antena monopolo de radiación isotrópica utilizada.

En la Figura 3 se registra la intensidad de la señal recibida en un recorrido en línea recta.

Tabla 7. Medición de la cobertura por llamada y sujeción a la red.

\begin{tabular}{|c|l|}
\hline ALCANCE DE COBERTURA $(\mathrm{m})$ & \multicolumn{1}{c|}{ CASO } \\
\hline 45 & Por sujeción a la red \\
\hline 19,25 & Por pérdida de llamada \\
\hline
\end{tabular}

Tabla 8. Medición de la cobertura por llamada y sujeción a la red.

\begin{tabular}{|c|c|c|}
\hline $\begin{array}{c}\text { DISTANCIA AL } \\
\text { ORIGEN [m] }\end{array}$ & $\begin{array}{c}\text { INTENSIDAD DE LA SEÑAL } \\
{[\mathrm{dBm}]}\end{array}$ & $\begin{array}{c}\text { DIRECCIÓN DE LA } \\
\text { MEDIDA }\end{array}$ \\
\hline 19,1 & -73 & SW \\
\hline 20 & -73 & SE \\
\hline 19,1 & -73 & NW \\
\hline
\end{tabular}


Figura 3. Medición de la intensidad de señal recibida en el MS para interiores.

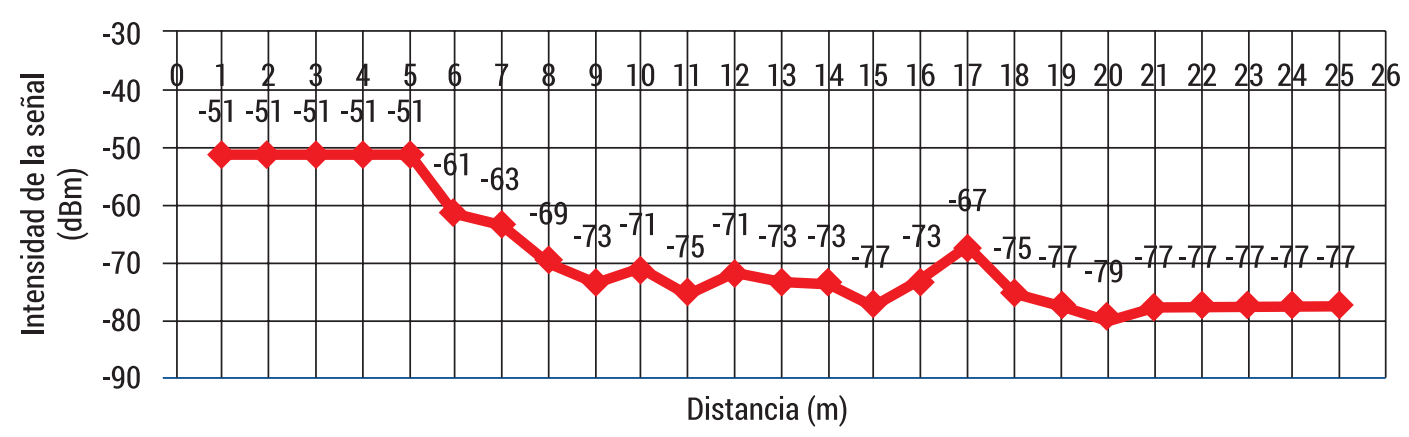

El alcance de la red GSM bajo los parámetros de diseño y el resultado obtenido difieren en un $6,8 \%$ de la distancia estimada utilizando el modelo Okumura-Hata. Las mediciones obtenidas en interiores a una distancia de 19 y 21 metros registran niveles de señal recibida de $-77 \mathrm{dBm}$ y $-73 \mathrm{dBm}$ respectivamente.

\subsection{FIABILIDAD Y CALIDAD}

Utilizando los reportes generados por la herramienta Asterisk se obtiene para la primera prueba 206 llamadas entre 7 MS, en la segunda prueba 47 llamadas entre 3 MS y la última prueba entre una MS y el SP en espacio libre. Los resultados de estas pruebas se muestran con los indicadores de desempeño KPI en la Tabla 9.

En laprimeraprueba, la probabilidad de bloqueo total fue del $12,1 \%$ entre llamadas fallidas y rechazadas. Sin embargo, ponderando solo las llamadas rechazadas, esta probabilidad de bloqueo resultante fue del $2 \%$. En cuanto a calidad, se otorgó una valoración subjetiva de 1 a 5 (donde 5 es muy buena calidad) para el audio. De las 66 llamadas contestadas se obtuvo un promedio de valoración de 4,0 entre las dos pruebas, siendo de menor calidad cerca del límite del alcance de la red a los 20m.
Tabla 9. Tráfico en el PBX Asterisk sobre la USRP1.

\begin{tabular}{|l|c|}
\hline ESTADÍSTICAS DE LLAMADAS DE VOZ & KPI \\
\hline Primera prueba & $88 \%$ \\
\hline Tasa de llamadas exitosas & $2,0 \%$ \\
\hline Tasa de llamadas rechazadas & $53 \%$ \\
\hline $\begin{array}{l}\text { Tiempo de establecimiento de la llamada } \\
\text { de voz < } 10 \text { s }\end{array}$ & 3,89848 \\
\hline Marcador promedio de opinión (MOS) & $83 \%$ \\
\hline Segunda prueba & $1.5 \%$ \\
\hline Tasa de llamadas exitosas & $88 \%$ \\
\hline Tasa de llamadas rechazadas & 4.08125 \\
\hline $\begin{array}{l}\text { Tiempo de establecimiento de la llamada } \\
\text { de voz < } 10 \text { s }\end{array}$ & $93 \%$ \\
\hline Marcador promedio de opinión (MOS) & $0 \%$ \\
\hline Prueba en espacio libre & $90 \%$ \\
\hline Tasa de llamadas exitosas & 4.1677 \\
\hline Tasa de llamadas rechazadas & $\begin{array}{l}\text { Tiempo de establecimiento de la llamada } \\
\text { de voz < } 10 \text { s }\end{array}$ \\
\hline Marcador promedio de opinión (MOS) & \\
\hline
\end{tabular}

\subsection{TRÁFICO}

Con la aplicación SIPP de Linux se generó un tráfico de llamadas de duración determinadas. Con la herramienta de Ubuntu "vmstat" severificó que la CPU se encontrara al $100 \%$ de capacidad (idle en $0 \%$ ). El PBX Asterisk se congestionó al alcanzar las 200 llamadas en paralelo, debido al acceso al medio de audio usado para realizar la prueba. En este estado, se determinó un uso de la CPU del $76 \%$ (id=24\%, inactividad). 
Tabla 10. Marcadores o contadores de los resultados arrojados por el registro del PBX Asterisk.

\begin{tabular}{|l|c|}
\hline MARCADORES DE LLAMADAS DE VOZ & RESULTADOS \\
\hline Primera prueba & 206 \\
\hline Número de intentos de llamada & 181 \\
\hline Número de llamadas exitosas & 22 \\
\hline Número de llamadas fallidas & 3 \\
\hline Número de llamadas rechazadas & 10,52913 \\
\hline Promedio duración de la llamada (s) & 2,5 \\
\hline Promedio tiempo de establecimiento de la llamada (s) & 3,898485 \\
\hline Marcador promedio de opinión (MOS de 0 a 5) & 66 \\
\hline Total de muestras del marcador promedio de opinión & 47 \\
\hline Segunda prueba & 39 \\
\hline Número de intentos de llamada & 2 \\
\hline Número de llamadas exitosas & 6 \\
\hline Número de llamadas fallidas & 7,213592233 \\
\hline Número de llamadas rechazadas & 6,787234043 \\
\hline Promedio duración de la llamada (s) & 4,08125 \\
\hline Promedio tiempo de establecimiento de la llamada (s) & 32 \\
\hline Marcador promedio de opinión (MOS de 0 a 5) & 44 \\
\hline Total de muestras del marcador promedio de opinión & 41 \\
\hline Prueba en espacio libre & 3 \\
\hline Número de intentos de llamada & 0 \\
\hline Número de llamadas exitosas & 34,72727273 \\
\hline Número de llamadas fallidas & 3,14893617 \\
\hline Número de llamadas rechazadas & 4,167742 \\
\hline Promedio duración de la llamada (s) & 31 \\
\hline Promedio tiempo de establecimiento de la llamada (s) & \\
\hline Marcador promedio de opinión (MOS de 0 a 5) & \\
\hline Total de muestras del marcador promedio de opinión & \\
\hline
\end{tabular}

El sistema soportó entre 200 y 263 llamadas en paralelo a una tasa de 10 llamadas por segundo bajo las características del hardware empleado. El sistema soportó 7 MS y un SP a la vez con su tráfico generado.

Utilizando las tablas de Erlang B en [29], se obtuvo un tráfico soportado por la USRP1 de 5,95 Erlang para una probabilidad de bloqueo del 12,1\%. Estas pruebas se realizaron con 7 MS y un SP. Esto implica que por cada línea se usan 0,744 Erlang, generando 9 llamadas por hora por cada línea con una duración de 5 minutos. Para el caso del uso de la probabilidad de bloqueo del $2 \%$ se obtiene un tráfico de 3,6 Erlang.

\section{CONCLUSIONES}

En el artículo se muestra la flexibilidad de los modelos de propagación ante diferentes escenarios, siendo más efectivo el modelo Walfisch-Ikegami para los casos donde se tiene información precisa sobre el entorno en cuanto a sus características topográficas. Sin embargo, para el cálculo de la cobertura de la red de emergencia GSM instalada se utilizó 
el modelo de propagación para interiores Okumura-Hata, arrojando un alcance máximo de 21 metros (sin amplificadores), este modelo incluyó las condiciones de zonas urbanas, interiores como edificios y materiales de construcción; siendo más cercano a las condiciones de prueba en comparación con los modelos de Friis y Walsfisch-Ikegami.

El tráfico soportado por la USRP1 mantiene una probabilidad de bloqueo del $2 \%$, al obtener un tráfico de 3,6 Erlang. Esto es un tráfico por línea de 0,45 Erlang, generando así 5 llamadas por hora por línea con una duración de 5 minutos.

\section{REFERENCIAS BIBLIOGRÁFICAS}

[1] Natalizio, E., Loscri, V. \& Aloi, G. (2010). The practical experience of implementing a GSM BTS through Open Software/ Hardware. Applied Sciences in Biomedical and Communication Technologies (ISABEL), pp.1-5.

[2] Fähnle, M. (2010). Software-Defined Radio with GNU Radio and USRP / 2 Hardware Frontend : Setup and FM / GSM Applications (Tesis de pregrado inédita). Hochschule Ulm University of Applied Sciences, Ulm, Alemania.

[3] Natalizio, E., Aloi, G., Loscri, V., Paoli, N. \& $\mathrm{N}, \mathrm{B}$. (2010). The practical experience of implementing a GSM BTS through open software/hardware. Applied Sciences in Biomedical and Communication Technologies (ISABEL), 23, pp.5-9.

[4] Mpala, J. \& Van Stam, G. (2012). Open BTS, a GSM experiment in rural Zambia. Africomm, Yaounde, Cameroon, pp.1-9.
[5] Dhananjay, A. \& Tierney, M. (2011). WiRE: a new rural connectivity paradigm. ACM SIGCOMM Computer Communication Review, 41(4), pp.462-463.

[6] Cabral, M., Almeida, I., Melo, C. \& Klautau, A. (2009). Low-cost GSM telephony in the Amazon region based on opensource / open-hardware projects. 2009 IEEE Latin-American Conference on Communications, pp.1-6.

[7] Hashem, I. E., ElShaarany, A., Abdelbarr, A. Y., Hassan, E. S. \& Fikri, M. (2012). Implementing an Enhanced base station using the software defined radio (CUOPENBTS). 2012 2nd International Conference on Advances in Computational Tools for Engineering Applications (ACTEA), pp.39-42.

[8] Van Stam, G. \& Mpala, J. (2013). Open BTS, a GSM experiment in rural Zambia. En e-Infrastructure and e-Services for Developing Countries. Berlín, Alemania: Ed. Springer, pp.65-73.

[9] Tsou, T. \& Cooper, T. (2012). Development of an open-source GSM femtocell and integrated core infrastructure. MILITARY COMMUNICATIONS CONFERENCE, pp.1-6.

[10] Cooper, T. A., Reed, J. H. \& Clancy, T. C. (2012). Integration of Open-Source GSM Networks Integration of OpenSource GSM Networks. Virginia, EE.UU.: Virginia Polytechnic Institute and Sate University.

[11] Liao, S. \& Bao, L. (2009). Implementing a base station using the $\mathrm{sdr}$ platform for coexistence of heterogeneous 
wireless systems. SDR Forum Technical Conference and Product Exposition, no. 0725914, pp.1-4.

[12] Bai, Y., Du, W., Ma, Z. \& Shen, C. (2010). Emergency communication system by heterogeneous wireless networking. Wireless Communications, Networking and Information Security (WCNIS), pp.488-492.

[13] Ochoa, S.F. \& Santos, R. (2013). Humancentric wireless sensor networks to improve information availability during urban search and rescue activities. Information Fusion, pp.9-11.

[14] Song, W., Wang, F. \& Dai, J. (2010). A Emergency Communication System based on WMN in underground mine. 2010 International Conference on Computer Application and System Modeling (ICCASM 2010), pp. V4-624-V4-627.

[15] Lien, Y.-N., Chi, L.-C. \& Huang, C.-C. (2010). A Multi-hop Walkie-Talkie-Like Emergency Communication System for Catastrophic Natural Disasters. 2010 39th International Conference on Parallel Processing Workshops, pp.527-532.

[16] Lien, Y.-N., Chi, L.-C. \& Shaw, Y.-S. (2009). A Walkie-Talkie-Like Emergency Communication System for Catastrophic Natural Disasters. 2009 10th International Symposium on Pervasive Systems, Algorithms, and Networks, pp.309-314.

[17] Hong, F., Yi, T., Zhao, H. \& Xin, B. (2009). Developing Test System of Ad Hoc Network for Emergency Communications. 2009 WRI World Congress on Computer Science and Information Engineering, pp.5-9.
[18] Shao, Z., Liu, Y., Wu, Y. \& Shen L. (2011). A Rapid and Reliable Disaster Emergency Mobile Communication System via Aerial Ad Hoc BSNetworks. Wireless Communications, Networking and Mobile Computing (WiCOM), no. 2007, pp.1-4.

[19] Gadallah, Y. \& Serhani, M. A. (2011). A WSN-driven service discovery technique for disaster recovery using mobile ad hoc networks. 2011 IFIP Wireless Days (WD), pp.1-5.

[20] Ahmed, M. \& Mansor, A.M. (2008). CPU dimensioning on performance of Asterisk VolP PBX. Proceedings of the 11th communications and networking simulation symposium, pp.139-146.

[21] Montoro, P. \& Casilari, E. (2009). A Comparative Study of VolP Standards with Asterisk. 2009 Fourth International Conference on Digital Telecommunications, pp.1-6.

[22] Gnuradio. (s.f.). OpenBTS. Recuperado en julio de 2014, de http://gnuradio. org/redmine/projects/gnuradio/wiki/ OpenBTSBackground.

[23] Ettus Research TM. (s.f.). Recuperado el 20 de julio de 2013, de www.ettus.com.

[24] ETSI (European Telecommunications Standards Institute). (1996). ETSI TCSMG ; 'GSM Technical Specification. GSM 05.05' phase 2+ Radio transmission and reception. Francia: ETSI.

[25] Cárdenas Valderrama, S. (2011). Dimensionamiento e implementación de una red cerrada de comunicaciones de emergencia a traves de la emulación 
de un sistema de conmutación de comunicaciones móviles celulares en la banda GSM (Tesis de pregarado inédita). Universidad Distrital Francisco José de Caldas, Bogotá, Colombia.

[26] Sah, N. (2005). ImpactofClutterson Quality of Service in Mobile Communication Using Walfisch-Ikegami Propagation Model. pp.290-294.

[27] Jeong, M. \& Lee, B. (2001). Comparison between path-loss prediction models for wireless telecommunication system design. IEEE Antennas and Propagation Society International Symposium. 2001
Digest. Held in conjunction with: USNC/ URSI National Radio Science Meeting (Cat. No.01CH37229), 2(8), pp.186189.

[28] Sarkar, T. K., Ji, Z., Kim, K., Medour, A. \& Salazar-Palma, M. (2003). A Survey of Various Propagation Models for Mobile Communication. Antennas and Propagation Magazine, IEEE, 45(3), pp.51-82.

[29] WestbayEngineers.(s.f.). Telecomunications software and services. Recuperado el 20 de julio de 2013, de http://www.erlang. com/calculator/erlb/ 